\title{
Lost in Translation
}

\author{
Nasia Safdar, MD; Dennis G. Maki, MD
}

Bloodstream infection (BSI) remains the most important infectious complication of vascular access and is associated with prolonged hospital stay ${ }^{1-4}$ and with greatly increased treatment $\operatorname{costs}^{1,2,4}$ and attributable mortality. ${ }^{1-3,5}$ Prevention of intravascular device-related (IVDR) BSI is fundamental to the patient safety movement, especially for patients requiring long-term vascular access for chemotherapy, parenteral nutrition, or hemodialysis. This issue of the journal contains 5 articles on the subject of healthcare-associated BSI: Warren et al. ${ }^{6}$ discuss the formidable challenges in translation of evidence-based medicine to evidence-based practice for prevention of IVDR BSI; Braun et al. ${ }^{7}$ compare the effect of using differing methods to ascertain rates of healthcare-associated $\mathrm{BSI}$; Maragakis et al. ${ }^{8}$ describe a very troubling increased incidence of BSI in association with a widely used commercial needleless connector; Menyhay and Maki' report the results of a simulation study of the effectiveness of conventional alcohol preparation of needleless luer-activated valve connectors with $70 \%$ alcohol and the efficacy of a novel antiseptic-barrier cap; and Huang et al. ${ }^{10}$ assess the utility of 2 blood cultures for diagnosis of BSI caused by coagulase-negative staphylococci (CNS) in neonates.

In recent years, a number of randomized controlled trials of preventive strategies for IVDR BSI have been conducted, and measures such as use of maximal barrier precautions for intravascular device insertion ${ }^{11}$ and use of chlorhexidine for cutaneous antisepsis ${ }^{12}$ have been shown unequivocally to greatly reduce the risk of IVDR BSI. It has also become apparent, however, that rigorous proof of efficacy, although necessary, is unfortunately not sufficient for these and other proven control measures to become an integral part of clinical practice. ${ }^{13,14}$ Much attention has focused on the factors that hinder implementation of evidence-based guidelines and that must be surmounted before these guidelines can be consistently incorporated into clinical practice to improve patient care. ${ }^{15-17}$ A number of evidence-based guidelines for the prevention of IVDR BSI have been published; the most recent was published by the Centers for Disease Control and Prevention (CDC) Healthcare Infection Control Practices Advisory Committee (HICPAC) in 2002. ${ }^{18}$

In this issue, Warren et al. ${ }^{6}$ report the findings of a survey undertaken to determine whether key evidence-based recommendations in the 1996 CDC guideline for prevention of $\mathrm{BSIs}^{18 a}$ that addressed short-term intravascular devices, including central venous catheters (CVCs) and peripherallyinserted CVCs, have been adopted by US hospitals. The authors surveyed 10 hospitals associated with the CDC Prevention Epicenters Program, all academic tertiary-care centers. Although the response rate was only $57 \%$ for the 25 intensive care units surveyed, and although it is unlikely that these academic centers are representative of US acute-care hospitals in general, the findings are disturbing. As of April 2002 , only $28 \%$ of the units that responded had a policy regarding the use of maximal sterile barrier precautions at the time of CVC insertion, and only half had a formal training program for aseptic and safe insertion of CVCs. Despite the fact that multiple studies have shown routine replacement of CVCs has no benefit for prevention of infection, in $16 \%$ of the intensive care units, catheters were still being routinely replaced, with the goal of preventing CVC-associated BSIs. The study does not provide data regarding the rates of IVDR BSI in the units surveyed, nor do we know to what extent the policies reflect actual clinical practice. Nonetheless, the variability among units noted by Warren et al. ${ }^{6}$ indicates that greater standardization and more uniform application of evidence-based guidelines is needed.

It must be emphasized that this survey was conducted 3 and a half years ago, and we think it is highly likely that the findings are no longer applicable to current hospital practices. We believe that the Joint Commission on Accreditation of Healthcare Organizations (JCAHO) care bundle (ie, group of best practices) ${ }^{19}$ and the Institute of Healthcare Improvement "Save 100,000 Lives" campaign ${ }^{20}$ have had a powerful effect on implementation of infection control measures for prevention of IVDR BSI in hospitals. Nearly all of our sister hospitals in southern Wisconsin have ongoing programs that focus on the JCAHO care bundle for prevention of IVDR BSI; the University of Wisconsin Hospital was surveyed by JCAHO in October 2005, and the surveyors specifically sought hard evidence that our institutional programs for protecting patients against IVDR BSI addressed most of the issues contained in the survey.

Drs. Safdar and Maki are from the Section of Infectious Diseases, Department of Medicine, University of Wisconsin Medical School, Madison, Wisconsin.

Received December 6, 2005; accepted December 6, 2005; electronically published January 6, 2006.

Infect Control Hosp Epidemiol 2006; 27:3-7

(C) 2006 by The Society for Healthcare Epidemiology of America. All rights reserved. 0195-9417/2006/2701-0002\$15.00. 
Exploring the reasons for the variability in catheter insertion and in care policy and practice was not an objective of the study by Warren et al., ${ }^{6}$ but there is an urgent need to better understand the barriers that impede effective translation of evidence-based guidelines into practice. Rubinson et al. ${ }^{21}$ performed a cross-sectional survey of 1000 physicians, with the goal of identifying and characterizing self-reported barriers to implementation of important evidence-based recommendations for prevention of IVDR BSI, on the basis of the 1996 CDC guideline. ${ }^{18 a}$ They found that high outcome expectancy was associated with greater adherence to recommendations but that awareness of the guideline per se did not influence adherence. The challenge of translating scientific evidence into clinical practice indicates that promulgation of a guideline must be supplemented by an intensive effort to assure a high level of adherence. Barriers to adherence may differ from institution to institution, but a multifaceted, systems-based approach, with a strong institutional commitment, has been shown to be highly effective for prevention of IVDR BSI. ${ }^{22}$

To determine the magnitude of the problem of IVDR BSI and to gauge the success of interventions for prevention, accurate measurement of BSI rates is essential, especially in light of legislation in many states that now mandates public reporting of rates of healthcare-associated infection. ${ }^{23}$ Rates of healthcare-associated BSI may be calculated in a number of ways: using either clinical or administrative data, using either hospital-wide or unit-specific data, with or without data on the source of BSI (primary or secondary), and with or without application of risk adjustment. In this issue, Braun et al. ${ }^{7}$ report the findings of a multiple-center observational study that undertook to measure BSI rates and used a variety of definitions and data collection approaches to ascertain whether differences in measurement affected the BSI rates found and the rankings of the hospitals. The authors show that differing denominator data, definitions of BSI, and methods of risk adjustment greatly affect the BSI rates derived. Therefore, benchmarking between institutions may be very misleading-even meaningless-if the methods of rate calculation are not uniform. These results highlight the urgent need to standardize definitions, methods of data collection, and methods of rate calculations for IVDR BSI and all reportable healthcare-associated infections across all reporting institutions.

Needleless connectors were developed, in response to demands for enhanced safety for healthcare workers, to prevent needlestick injuries and are integral components of infusion systems in use across North America. The 3 major design categories of needleless connectors are the split septum design, the luer-activated valve, and the luer valve with positive displacement. Numerous commercial products are available within each category. Although needleless connectors, when properly used, clearly reduce the risk of needlestick injuries associated with accessing the intravascular device or injection ports, ${ }^{24-27}$ a handful of reports published over the past decade have raised niggling concerns about a potential increased risk of iatrogenic BSI associated with their use. ${ }^{28-32}$ Most of these studies have been retrospective and uncontrolled, and suboptimal usage of the device, rather than the device itself, may have been responsible for the increased incidence of BSI in some settings. The few randomized trials comparing needleless connection devices with standard connectors have found relative reductions in BSI rates with the use of the needleless device under study, ranging from $84 \%$ to $86 \%,{ }^{33,34}$ and the most recent CDC HICPAC guideline on prevention of IVDR BSI concludes that, "when used properly, needleless devices do not adversely affect the incidence of BSI."18(p11)

In this issue of the journal, Maragakis et al. ${ }^{8}$ describe a sharp increase in BSI rates following introduction of a new needleless connector with a positive-pressure mechanical valve designed to prevent reflux of blood into the catheter. BSI rates increased from a baseline rate (pooled across all ICUs) of 1.5 cases per 1000 catheter-days to 2.4 cases per 1000 catheter-days $(P=.03)$. A shift in the microbial profile of IVDR BSI was also seen, with a much higher proportion of cases caused by gram-negative bacilli (an increase from $17.7 \%$ to $28.1 \%$ ) or multiple organisms (an increase from $6.5 \%$ to $14 \%$ ). Rates of IVDR BSI fell back to baseline levels after the institution discontinued use of the new needleless connector and resumed use of the needleless connector used previously, which did not have a positive-pressure mechanical valve.

As Maragakis et al. ${ }^{8}$ point out, their study was retrospective and uncontrolled, thus making it difficult to be certain that the new needleless connector was the causal factor in the strikingly increased incidence of BSI they observed. Details regarding the type of training and education provided to healthcare staff before introduction of the new connector are not provided; as prior studies have suggested, suboptimal use may be one reason for an intravascular device to be associated with an increased risk of BSI. Although the authors state that their investigations did not uncover major violations of infection control policies at the time of the BSI outbreak, it is unclear whether a formal procedural review was undertaken.

In the study of Maragakis et al., ${ }^{8}$ the administration set and the end caps were changed every 96 hours. Until recently, the manufacturer of the positive-pressure needleless connector did not provide recommendations regarding the frequency of connector change. The manufacturer now recommends that the connector be changed every 72 hours or 100 activations, whichever comes first. In an earlier study of IVDR BSI in the home-care setting, Do An et al..$^{28}$ found that altering the frequency of end cap change from once weekly to once every 2 days greatly reduced the risk of BSI, suggesting that the mechanism for BSI associated with use of the device involves contamination from the point of access. It is essential to remain vigilant regarding any undesirable consequences associated with use of needleless connectors, which are now nearly universally used in US hospitals. Future studies exploring this association should endeavor to confirm that a 
needleless device is being used properly, to better ascertain whether an increased risk of BSI is causally related to the design of the device itself or to the way the device is used.

Although needleless connectors and injection ports are recognized sites of access for microbial contamination, no national standard exists defining the best and recommended form of antiseptic preparation for prevention of microbial entry when the needleless connector or injection port is accessed. There are, to our knowledge, no clinical trials that have prospectively examined the efficacy of various approaches to disinfection for prevention of CVC-related BSI. The 'most recent CDC HICPAC guideline has recommended that vascular catheter hubs, needleless connectors, and injection ports be disinfected before being accessed, and this recommendation is classified as category $1 \mathrm{~B}^{18}$; but the guideline does not recommend any specific disinfection agent or protocol. In most US healthcare centers, the practice is to disinfect catheter hubs, needleless connectors, and injection ports by swabbing of the membranous septum with $70 \%$ isopropyl alcohol; however, the study of Menyhay and Maki ${ }^{9}$ and limited prior studies ${ }^{35-37}$ show that this may not reliably eliminate septal surface contamination. It is noteworthy that novel technological approaches to preventing contamination of CVC administration-set connections by use of a povidone-iodine "shield" 35 or a novel antiseptic-containing connector ${ }^{36,37}$ have been shown to reduce rates of CVC-associated BSI in studies done in Europe in settings where there was a very high baseline rate of CVC-associated BSI.

Also in this issue of the journal, Menyhay and Maki' report the results of a simulation study of the effectiveness of conventional preparation of needleless luer-activated valve connectors with $70 \%$ alcohol and the efficacy of a novel antiseptic-barrier cap that, when threaded onto a luer-adaptable connector, rapidly sterilized a surface contaminated with $\sim 10^{5}$ colony forming units of Enterococcus faecalis. ${ }^{9}$ Of 60 contaminated connectors inserted after application of the novel antiseptic cap for 10 minutes, only $1(1.6 \%)$ showed any microbial growth, compared with 15 control devices that showed heavy bacterial growth $(P<.001)$. This was an in vitro study, and this novel antiseptic device needs to be tested in clinical trials to determine its efficacy. Novel technology is probably the most effective way to prevent contamination at the point of access and should be studied further.

Coagulase-negative staphylococci are a major cause of healthcare-associated infection, ${ }^{38-41}$ especially in immunocompromised patients and neonates and, according to data from the National Nosocomial Infections Surveillance system, are the foremost cause of healthcare-associated BSI in the United States. ${ }^{42}$ They are also major components of the normal skin and mucosal microflora, and in clinical practice it is a major challenge to distinguish true bacteremia caused by coagulase-negative staphylococci from contamination that occurs at the time when blood is obtained for culture. The lack of an adequate reference standard for definition of true bacteremia-which is defined variably as a patient's receipt of antibiotics for more than 4 days, or an explicit note in the medical record that the physician diagnosed a true bacteremia, or the CDC's surveillance criteria for primary BSImakes it difficult to compare methods that distinguish between contamination and bacteremia. Detection of genes putatively associated with virulence has yielded conflicting results, ${ }^{43-46}$ and the role of this method in clinical decision making appears to be limited. Strain typing and antibiogram analysis of isolates also do not provide rapid or very useful adjunctive information to aid in clinical decision making. A recent study in children showed that the use of the time to positivity of blood culture for growth of coagulase-negative staphylococci in a continuously monitored blood culture system (Bactec; Becton Dickinson) was useful in distinguishing between contamination and bacteremia; a time to positivity of $\leqslant 15$ hours had a positive predictive value of $84 \%$ for diagnosis of true bacteremia. ${ }^{47}$

One important, widely accepted criterion for true bacteremia is the isolation of coagulase-negative staphylococci from multiple blood cultures. Use of this criterion presumes that the isolates represent a single clone of the pathogen-which may or may not be valid, since all persons are normally colonized by multiple clones of coagulase-negative staphylococci. If blood samples for culture are collected from different body sites, the contamination of each blood sample during the collection process is an independent, chance event. Therefore, multiple cultures positive for coagulase-negative staphylococci are less likely to represent contamination than true bacteremia. Comparing strain clonality with the clinical criteria for BSI, Seo et al. ${ }^{48}$ found that patients with 3 or more positive blood culture results were more likely to have samestrain bacteremia than were patients with only 2 positive culture results ( 11 of 15 [73\%] vs 8 of 27 [30\%]; $P=.006$ ). Unfortunately, there was poor agreement between strain relatedness and clinical definitions of bacteremia. ${ }^{48}$ Using mathematical modeling, Tokars ${ }^{49}$ found that the positive predictive value for bacteremia was $55 \%$ for 1 positive culture result for a single sample cultured, but increased to $98 \%$ for 2 positive culture results if both blood samples were obtained percutaneously.

In line with current evidence, Huang et al. ${ }^{10}$ report in this issue of the journal that, in neonates with clinical sepsis, 12 of 13 cultures of percutaneously obtained, paired blood samples yielded the same strain, a finding that suggests infection rather than contamination. The authors do not provide data on the courses of the patients in the intensive care unit or the courses of a comparator group of patients from whom only a single blood sample for culture was drawn. Moreover, they provide no reference standard for true bacteremia, such as a quantitative clinical scoring system. ${ }^{50}$

Finally, reducing the rate of contamination of blood cultures may best control the huge adverse clinical and economic impact of contamination of blood cultures with coagulasenegative staphylococci. Multiple prospective trials have found that chlorhexidine or tincture of iodine are much more ef- 
fective than povidone iodine for cutaneous antisepsis before drawing the blood sample, providing $50 \%$ lower rates of contamination, on average. ${ }^{51-55}$ Having a team of trained phlebotomists to collect all blood for culture, ${ }^{56}$ providing feedback to phlebotomists regarding contamination rates, ${ }^{57}$ and tracking of the blood-culture contamination rate $^{58}$ as a quality indicator are all practices that have been shown to lower contamination rates.

Address reprint requests to Dennis G. Maki, MD, University of Wisconsin Hospital and Clinics, 600 Highland Avenue, Madison, WI 53792 (dgmaki@medicine.wisc.edu).

\section{REFERENCES}

1. Arnow PM, Quimosing EM, Beach M. Consequences of intravascular catheter sepsis. Clin Infect Dis 1993; 16:778-784.

2. Pittet D, Tarara D, Wenzel RP. Nosocomial bloodstream infection in critically ill patients: excess length of stay, extra costs and attributable mortality. JAMA 1994; 271:1598-1601.

3. Collignon PJ. Intravascular catheter associated sepsis: a common problem. The Australian Study on Intravascular Catheter Associated Sepsis. Med J Aust 1994; 161:374-378.

4. Rello J, Ochagavia A, Sabanes E, et al. Evaluation of outcome of intravenous catheter-related infections in critically ill patients. Am J Respir Crit Care Med 2000; 162:1027-1030.

5. Smith RL, Meixler SM, Simberkoff MS. Excess mortality in critically ill patients with nosocomial bloodstream infections. Chest 1991; 100:164167.

6. Warren DK, Yokoe DS, Climo MW, et al. Preventing catheter-associated bloodstream infections: a survey of policies for insertion and care of central venous catheters from hospitals in the Prevention Epicenter Program. Infect Control Hosp Epidemiol 2006; 27:8-13 (in this issue).

7. Braun BI, Kritchevsky SB, Kusek L, et al. Comparing bloodstream infection rates: the effect of indicator specifications in the Evaluation of Processes and Indicators in Infection Control (EPIC) study. Infect Control Hosp Epidemiol 2006; 27:14-22 (in this issue).

8. Maragakis LL, Bradley KL, Song X, et al. Increased catheter-related bloodstream infection rates after the introduction of a new mechanical valve intravenous access port. Infect Control Hosp Epidemiol 2006; 27:67-70 (in this issue).

9. Menyhay SZ, Maki DG. Disinfection of needleless connectors and access ports with alcohol may not prevent microbial entry: the promise of a novel antiseptic barrier cap. Infect Control Hosp Epidemiol 2006; 27:2327 (in this issue).

10. Huang Y-C, Wang Y-H, Su L-H, Chou Y-H, Lien R-I, Lin T-Y. Determining the significance of coagulase-negative staphylococci identified in cultures of paired blood specimens from neonates by species identification and strain clonality. Infect Control Hosp Epidemiol 2006; 27:70-73 (in this issue).

11. Raad, II, Hohn DC, Gilbreath BJ, et al. Prevention of central venous catheter-related infections by using maximal sterile barrier precautions during insertion. Infect Control Hosp Epidemiol 1994; 15:231-238.

12. Chaiyakunapruk N, Veenstra DL, Lipsky BA, Saint S. Chlorhexidine compared with povidone-iodine solution for vascular catheter-site care: a meta-analysis. Ann Intern Med 2002; 136:792- 801.

13. Berenholtz SM, Pronovost PJ. Barriers to translating evidence into practice. Curr Opin Crit Care 2003; 9:321-325.

14. Maue SK, Segal R, Kimberlin CL, Lipowski EE. Predicting physician guideline compliance: an assessment of motivators and perceived barriers. Am J Manag Care 2004; 10:383-391.

15. Cabana MD, Rand CS, Powe NR, et al. Why don't physicians follow clinical practice guidelines? A framework for improvement. JAMA 1999; 282:1458-1465.

16. Pathman DE, Konrad TR, Freed GL, Freeman VA, Koch GG. The awareness-to-adherence model of the steps to clinical guideline compliance: the case of pediatric vaccine recommendations. Med Care 1996; 34:873889.

17. Davis DA, Thomson MA, Oxman AD, Haynes RB. Changing physician performance: a systematic review of the effect of continuing medical education strategies. JAMA 1995; 274:700-705.

18. O'Grady NP, Alexander M, Dellinger EP, et al. Centers for Disease Control and Prevention. Guidelines for the prevention of intravascular catheter-related infections. MMWR Recomm Rep 2002; 51(RR-10):1-29.

18a. Pearson ML. Guideline for the prevention of intravascular-device-related infections, Part I. Intravascular-device-related infections: an overview. Infect Control Hosp Epidemiol 1996; 24:262-277.

19. Joint Commission on Accreditation of Healthcare Organizations (JCAHO). Bundle for prevention of intravascular device-related bloodstream infections. Available at http://www.jcaho.org/. Accessed November 27, 2005.

20. Institute of Healthcare Improvement (IHI) "Save 100,000 Lives" campaign. IHI Web site. Available at http://www.ihi.org/IHI/Programs/Campaign/. Accessed November 27, 2005.

21. Rubinson $\mathrm{L}, \mathrm{Wu} \mathrm{AW}$, Haponik EF, Diette GB. Why is it that internists do not follow guidelines for preventing intravascular catheter infections? Infect Control Hosp Epidemiol 2005; 26:525-533.

22. Berenholtz SM, Pronovost PJ, Lipsett PA, et al. Eliminating catheterrelated bloodstream infections in the intensive care unit. Crit Care Med 2004; 32:2014-2020.

23. McKibben L, Horan T, Tokars II, et al. Guidance on public reporting of healthcare-associated infections: recommendations of the Healthcare Infection Control Practices Advisory Committee. Am J Infect Control 2005; 33:217-226.

24. Mendelson MH, Short LJ, Schechter CB, et al. Study of a needleless intermittent intravenous-access system for peripheral infusions: analysis of staff, patient, and institutional outcomes. Infect Control Hosp Epidemiol 1998; 19:401-406.

25. Lawrence LW, Delclos GL, Felknor SA, et al. The effectiveness of a needleless intravenous connection system: an assessment by injury rate and user satisfaction. Infect Control Hosp Epidemiol 1997; 18:175-182.

26. Skolnick R, LaRocca J, Barba D, Paicius L. Evaluation and implementation of a needleless intravenous system: making needlesticks a needless problem. Am J Infect Control 1993; 21:39-41.

27. Gartner K. Impact of a needleless intravenous system in a university hospital. J Healthc Mater Manage 1993; 11:44-46, 48-49.

28. Do AN, Ray BJ, Banerjee SN, et al. Bloodstream infection associated with needleless device use and the importance of infection-control practices in the home health care setting. I Infect Dis 1999; 179:442-448.

29. McDonald LC, Banerjee SN, Jarvis WR. Line-associated bloodstream infections in pediatric intensive-care-unit patients associated with a needleless device and intermittent intravenous therapy. Infect Control Hosp Epidemiol 1998; 19:772-777.

30. Cookson ST, Ihrig M, O'Mara EM, et al. Increased bloodstream infection rates in surgical patients associated with variation from recommended use and care following implementation of a needleless device. Infect Control Hosp Epidemiol 1998; 19:23-27.

31. Kellerman S, Shay DK, Howard J, et al. Bloodstream infections in home infusion patients: the influence of race and needleless intravascular access devices. J Pediatr 1996; 129:711-717.

32. Danzig LE, Short LJ, Collins K, et al. Bloodstream infections associated with a needleless intravenous infusion system in patients receiving home infusion therapy. JAMA 1995; 273:1862-1864.

33. Yebenes IC, Vidaur L, Serra-Prat M, et al. Prevention of catheter-related bloodstream infection in critically ill patients using a disinfectable, needle-free connector: a randomized controlled trial. Am J Infect Control 2004; 32:291-295.

34. Inoue $\mathrm{Y}$, Nezu R, Matsuda $\mathrm{H}$, et al. Prevention of catheter-related sepsis 
during parenteral nutrition: effect of a new connection device. JPEN $J$ Parenter Enteral Nutr 1992; 16:581-585.

35. Halpin DP, O'Byrne P, McEntee G, Hennessy TP, Stephens RB. Effect of a betadine connection shield on central venous catheter sepsis. $\mathrm{Nu}$ trition 1991; 7:33-34.

36. Inoue $\mathrm{Y}$, Nezu R, Matsuda $\mathrm{H}$, et al. Experimental study of hub contamination: effect of a new connection device: the I system. JPEN J Parenter Enteral Nutr 1992; 16:178-180.

37. Stotter AT, Ward $\mathrm{H}$, Waterfield AH, Hilton J, Sim AJ. Junctional care: the key to prevention of catheter sepsis in intravenous feeding. JPEN $J$ Parenter Enteral Nutr 1987; 11:159-162.

38. Souvenir D, Anderson DE, Jr., Palpant S, et al. Blood cultures positive for coagulase-negative staphylococci: antisepsis, pseudobacteremia, and therapy of patients. J Clin Microbiol 1998; 36:1923-1926.

39. Mutnick AH, Kirby JT, Jones RN. CANCER resistance surveillance program: initial results from hematology-oncology centers in North America. Chemotherapy Alliance for Neutropenics and the Control of Emerging Resistance. Ann Pharmacother 2003; 37:47-56.

40. Kloos WE, Bannerman TL. Update on clinical significance of coagulasenegative staphylococci. Clin Microbiol Rev 1994; 7:117-140.

41. Thylefors JD, Harbarth S, Pittet D. Increasing bacteremia due to coagulase-negative staphylococci: fiction or reality? Infect Control Hosp Epidemiol 1998; 19:581-589.

42. Richards MJ, Edwards JR, Culver DH, Gaynes RP. Nosocomial infections in medical intensive care units in the United States. National Nosocomial Infections Surveillance System. Crit Care Med 1999; 27:887-892.

43. Frebourg NB, Lefebvre S, Baert S, Lemeland JF. PCR-based assay for discrimination between invasive and contaminating Staphylococcus epidermidis strains. J Clin Microbiol 2000; 38:877-880.

44. Rohde H, Kalitzky M, Kroger N, et al. Detection of virulence-associated genes not useful for discriminating between invasive and commensal Staphylococcus epidermidis strains from a bone marrow transplant unit. $J$ Clin Microbiol 2004; 42:5614-5619.

45. Vandecasteele SJ, Peetermans WE, R RM, Rijnders BJ, Van Eldere J. Reliability of the ica, aap and atlE genes in the discrimination between invasive, colonizing and contaminant Staphylococcus epidermidis isolates in the diagnosis of catheter-related infections. Clin Microbiol Infect 2003; 9:114-119.

46. Galdbart JO, Allignet J, Tung HS, Ryden C, El Solh N. Screening for Staphylococcus epidermidis markers discriminating between skin-flora strains and those responsible for infections of joint prostheses. I Infect Dis 2000; 182:351-355.

47. Haimi-Cohen Y, Shafinoori S, Tucci V, Rubin LG. Use of incubation time to detection in BACTEC 9240 to distinguish coagulase-negative staphylococcal contamination from infection in pediatric blood cultures. Pediatr Infect Dis J 2003; 22:968-974.

48. Seo SK, Venkataraman L, DeGirolami PC, Samore MH. Molecular typing of coagulase-negative staphylococci from blood cultures does not correlate with clinical criteria for true bacteremia. Am J Med 2000; 109:697704.

49. Tokars JI. Predictive value of blood cultures positive for coagulase-negative staphylococci: implications for patient care and health care quality assurance. Clin Infect Dis 2004; 39:333-341.

50. Mahieu LM, De Dooy JJ, Cossey VR, et al. Internal and external validation of the NOSEP prediction score for nosocomial sepsis in neonates. Crit Care Med 2002; 30:1459-1466.

51. Calfee DP, Farr BM. Comparison of four antiseptic preparations for skin in the prevention of contamination of percutaneously drawn blood cultures: a randomized trial. J Clin Microbiol 2002; 40:1660-1665.

52. Champagne S, Fussell S, Scheifele D. Evaluation of skin antisepsis prior to blood culture in neonates. Infect Control 1984; 5:489-491.

53. Little JR, Murray PR, Traynor PS, Spitznagel E. A randomized trial of povidone-iodine compared with iodine tincture for venipuncture site disinfection: effects on rates of blood culture contamination. Am J Med 1999; 107:119-125.

54. Trautner BW, Clarridge JE, Darouiche RO. Skin antisepsis kits containing alcohol and chlorhexidine gluconate or tincture of iodine are associated with low rates of blood culture contamination. Infect Control Hosp Epidemiol 2002; 23:397-401.

55. Strand CL, Wajsbort RR, Sturmann K. Effect of iodophor vs iodine tincture skin preparation on blood culture contamination rate. JAMA 1993; 269:1004-1006.

56. Surdulescu S, Utamsingh D, Shekar R. Phlebotomy teams reduce bloodculture contamination rate and save money. Clin Perform Qual Health Care 1998; 6:60-62.

57. Gibb AP, Hill B, Chorel B, Brant R. Reduction in blood culture contamination rate by feedback to phlebotomists. Arch Pathol Lab Med 1997; 121:503-507.

58. Weinbaum FI, Lavie S, Danek M, Sixsmith D, Heinrich GF, Mills SS. Doing it right the first time: quality improvement and the contaminant blood culture. J Clin Microbiol 1997; 35:563-565. 